\title{
ESTUDO DO PADRÃO ALIMENTAR TARDIO EM OBESOS SUBMETIDOS À DERIVAÇÃO GÁSTRICA COM BANDAGEM EM Y-DE-ROUX: COMPARAÇÃO ENTRE HOMENS E MULHERES
}

\author{
LATE MEAL PATTERN IN OBESE PEOPLE AFTER BANDED ROUX-EN-Y GASTRIC \\ BYPASS: COMPARISION BETWEEN MALE AND FEMALE
}

\author{
Antonio Carlos Valezi, TCBC-PR ${ }^{1}$; Silvia Jamusse de Brito ${ }^{2}$; \\ Jorge Mali Junior, ACBC-PR²; Edivaldo Macedo de Brito, ACBC-PR ${ }^{3}$
}

\begin{abstract}
RESUMO: Objetivo: Avaliar o padrão alimentar caracterizando preferências e intolerâncias alimentares em pacientes submetidos à derivação gástrica com bandagem em Y-de-Roux frente às modificações decorrentes da capacidade gástrica reduzida e também se há diferenças entre homens e mulheres em relação à alimentação após cirurgia. Métodos: De julho de 2006 a julho de 2007 foi realizado um estudo transversal descritivo, de amostra por conveniência com 116 pacientes, após 12 meses de cirurgia de derivação gástrica com bandagem em Y-de-Roux, no ambulatório de Cirurgia Digestiva do Hospital Universitário da Universidade Estadual de Londrina. Foi aplicado um questionário abordando características demográficas, informações referentes ao hábito e padrão alimentar, presença de vômitos e sintomas de "dumping". As variáveis foram analisadas aplicando o teste exato de Fischer ou Qui-quadrado. Resultados: Dos 116 pacientes 91(78,4\%) eram mulheres e 25(21,5\%) homens. Não houve diferença significativa entre homens e mulheres quanto ao tempo das refeições, número de refeições, líquidos nas refeições e ingestão hídrica diária. Quanto ao consumo de alimentos, relato de vômitos e sintomas de "dumping" houve pequena diferença entre homens e mulheres, no entanto sem diferença significativa a não ser quanto à ingestão de frutas e sonolência significativamente mais freqüente em mulheres. Conclusão: Com o questionário proposto foi possível avaliar o padrão tardio de aceitação alimentar dos pacientes e constatar que existe diferença entre homens e mulheres nas variáveis analisadas, apenas para o consumo de frutas (Rev. Col. Bras. Cir. 2008; 35(6): 387-391).
\end{abstract}

Descritores: Alimentação; Obesidade mórbida; Comportamento Alimentar; Cirurgia bariátrica; Derivação Gástrica; Hábitos alimentares.

\section{INTRODUÇÃO}

A obesidade é uma doença metabólica de origem multifatorial, envolvendo fatores genéticos, endócrinos e ambientais ${ }^{1}$. O aumento do peso corpóreo associa-se a muitas comorbidades ${ }^{2,3}$. A obesidade determina ainda problemas psicológicos e sociais e diminuição da qualidade de vida ${ }^{4}$.

$\mathrm{O}$ tratamento conservador para obesidade é ineficaz em produzir perdas significativas do peso e apresenta elevado índice de recidiva ${ }^{5}$. A cirurgia é atualmente o tratamento mais eficaz para a redução de peso e manutenção dessa perda em pacientes com obesidade grave ${ }^{6}$. Dentre as várias técnicas cirúrgicas propostas para o tratamento da obesidade, a derivação gástrica com bandagem em Y-de-Roux tem sido a mais utilizada em nosso país ${ }^{7}$.

Recomenda-se, ao paciente operado, seguimento em longo prazo, pelo risco significativo de alterações nutricionais. $\mathrm{O}$ sucesso da cirurgia não deve ser medido apenas pela perda do peso, mas é influenciado pela qualidade da readequação alimentar. Muitos pacientes têm dificuldade nessa adaptação, fazendo opção por alimentos pouco nutritivos, porém bem tolerados. A orientação é indispensável à ingestão de dieta adequada nutricionalmente e correta para assegurar a perda de peso.

Estudos tem sido realizados ${ }^{8}$ com pacientes submetidos à cirurgia abordando as vantagens das diferentes técnicas cirúrgicas, morbidade e mortalidade pós-operatória, modificações dos exames bioquímicos, desaparecimento dos sintomas clínicos, análise da qualidade de vida, no entanto são poucos os estudos sobre o padrão alimentar a longo prazo. Não há estudos comparando os gêneros masculino e feminino em relação à aceitação alimentar pós-operatória.

O objetivo do presente estudo é avaliar o padrão alimentar caracterizando preferências e intolerâncias alimentares de pacientes submetidos à derivação gástrica com bandagem em Y-de-Roux e também avaliar se há diferenças entre homens e mulheres em relação à alimentação após a cirurgia.

\section{MÉTODO}

Realizou-se estudo transversal descritivo, qualitativo e quantitativo, de amostra por conveniência, de obesos

1. Professor Associado; Doutor do Departamento de Cirurgia da Universidade Estadual de Londrina- PR.

2. Pós-Graduando do Centro de Ciências da Saúde da Universidade Estadual de Londrina - PR.

3. Professor Adjunto; Mestre do Departamento de Cirurgia da Universidade Estadual de Londrina - PR.

Recebido em 25/06/2008

Aceito para publicação em 29/08/2008

Conflito de interesses: nenhum

Fonte de financiamento: nenhuma

Trabalho realizado no Hospital Universitário da Universidade Estadual de Londrina - PR. 
que foram submetidos à derivação gástrica com bandagem em Y-de-Roux após avaliação da equipe multiprofissional do serviço. Considerou-se como critério de inclusão, pacientes com 12 meses de pós-operatório e em seguimento por equipe multiprofissional no Ambulatório de Cirurgia do Aparelho Digestivo do Hospital. Universitário da Universidade Estadual de Londrina. Foram excluídos pacientes que tiveram qualquer complicação pós-operatória. A amostra constituiu-se de 116 pacientes avaliados entre julho de 2006 a julho de 2007. Idade variando de 20 a 65 anos.

Após informação e livre consentimento, foi aplicado aos pacientes, pelo mesmo pesquisador, questionário abordando características demográficas e informações referentes ao padrão alimentar, alimentos consumidos, número de refeições diárias, capacidade de consumir carnes, arroz, feijão, vegetais, frutas e derivados do leite, preferências e intolerâncias alimentares e ingestão hídrica. Foram questionados quanto à freqüência dos vômitos, sintomas de "dumping" e hábito intestinal. Solicitou-se aos pacientes, que descrevessem como se sentiam comparativamente à alimentação anterior à operação, em relação à diminuição do volume alimentar.

O estudo foi aprovado pelo Comitê de Ética em Pesquisa da Universidade Estadual de Londrina.

A amostra foi dividida em dois grupos: gênero masculino e feminino. As variáveis quantitativas foram expressas em média e as variáveis qualitativas em frequiência simples e percentual. A associação das variáveis qualitativas com o gênero masculino e feminino foi analisada aplicando o teste de qui-quadrado e nos casos com freqüência esperada menor do que cinco foi aplicado o teste exato de Fisher. Os testes foram realizados em nível de significância de $5 \%$ e processados no programa Epi Info versão 3 .

\section{RESULTADOS}

Dos 116 pacientes estudados, 25 (21,5\%) eram do gênero masculino e $91(78,4 \%)$ do gênero feminino. As características desses pacientes não diferiram quanto à idade, estatura, peso pré-operatório e IMC pré e pós-operatório.

O grau de escolaridade dos pacientes do gênero feminino não diferiu do masculino.

Os hábitos alimentares pesquisados dos homens e das mulheres submetidos à derivação gástrica não foram estatisticamente diferentes $(p>0,05)$. Os dados referentes ao, tempo gasto durante a refeição, número de refeições, consumo de líquidos durante as refeições e ingestão hídrica diária estão apresentados na tabela 1 . O padrão de alimentação dos pacientes no período pós-operatório conforme aceitação e tolerância estão representados na tabela 2. Das variáveis analisadas somente a freqüência do consumo de frutas diferiu entre homens e mulheres $(p=0,03)$. Foi considerado pouco freqüente quando o paciente referia que consumia o tipo de alimento três vezes ou menos por semana. Os pacientes referiram ingerir cerveja, refrigerantes, "chips", chocolates e sorvetes. Os homens (44\%) preferiram refrigerantes, cervejas e salgadinhos tipo "chips". As mulheres $(50,5 \%)$ chocolates, refrigerantes e sorvetes. Os pacientes foram questionados sobre vômitos relacionados à alimentação se espontâneos ou induzidos (Tabela 3).

\section{DISCUSSÃO}

A qualidade de vida inclui diversos parâmetros. A capacidade de comer e apreciar alimentos de forma variada, de maneira isolada ou em sociedade, certamente

Tabela 1 - Tempo, número e líquidos nas refeições e ingestão hídrica diária.

\begin{tabular}{|c|c|c|c|c|c|c|c|}
\hline \multirow{3}{*}{ Hábitos Alimentares } & \multicolumn{4}{|c|}{ Sexo } & & & \multirow[t]{3}{*}{ Valor de $p$} \\
\hline & \multicolumn{2}{|c|}{ Feminino } & \multicolumn{2}{|c|}{ Masculino } & \multicolumn{2}{|c|}{ Total } & \\
\hline & $\mathbf{n}$ & $\%$ & $\mathbf{n}$ & $\%$ & $\mathbf{n}$ & $\%$ & \\
\hline \multicolumn{8}{|l|}{ Tempo das Refeições } \\
\hline Menos de 15 min & 37 & 40,7 & 8 & 32,0 & 45 & 38,8 & 0,72 \\
\hline 15 a $30 \mathrm{~min}$ & 39 & 42,9 & 12 & 48,0 & 51 & 44,0 & 0,74 \\
\hline Mais de $30 \mathrm{~min}$ & 15 & 16,5 & 5 & 20,0 & 20 & 17,2 & 0,77 \\
\hline \multicolumn{8}{|l|}{ Número de Refeições } \\
\hline Três ou menos & 12 & 13,2 & 1 & 4,0 & 13 & 11,2 & 0,72 \\
\hline Quatro ou cinco & 51 & 56,0 & 21 & 84,0 & 72 & 62,1 & 0,73 \\
\hline Mais de cinco & 28 & 30,8 & 3 & 12,0 & 31 & 26,7 & 0,77 \\
\hline \multicolumn{8}{|l|}{ Líquidos nas Refeições } \\
\hline Sim & 31 & 34,1 & 10 & 40,0 & 41 & 35,3 & 0,58 \\
\hline Não & 60 & 65,9 & 15 & 60,0 & 75 & 64,7 & 0,69 \\
\hline \multicolumn{8}{|c|}{ Ingestão Hídrica Diária } \\
\hline Até 500 & 30 & 33,0 & 8 & 32,0 & 38 & 32,8 & 0,98 \\
\hline 500 a 1000 & 51 & 56,0 & 14 & 56,0 & 65 & 56,0 & 0.99 \\
\hline Mais de 1000 & 10 & 11,0 & 3 & 12,0 & 13 & 11,2 & 0,98 \\
\hline
\end{tabular}

Valor de p: nenhuma das variáveis apresentou diferença significativa. 
Tabela 2 - Padrão (aceitação e tolerância) da alimentação após cirurgia.

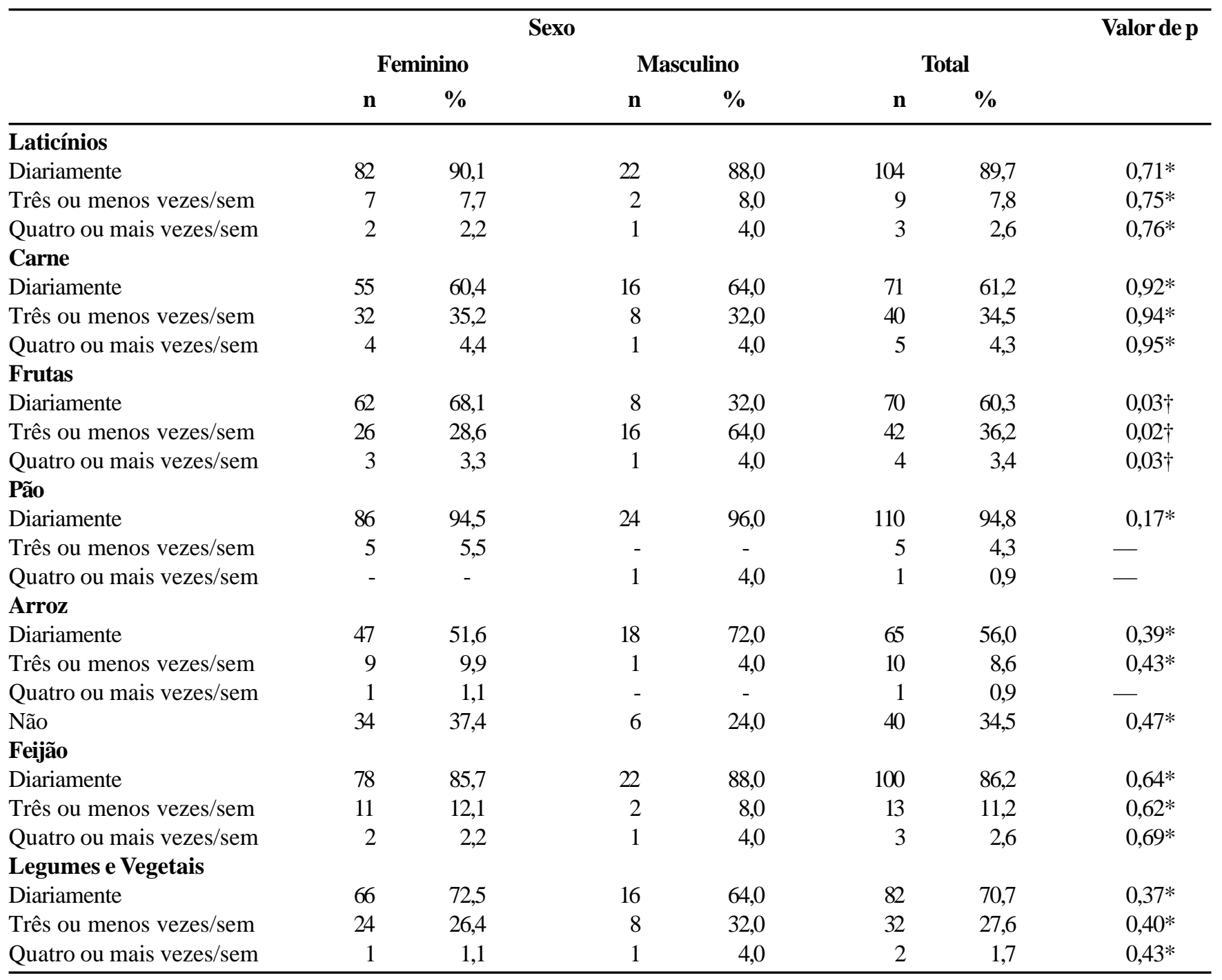

Vezes/sem = vezes na semana.

* valor de p do teste de qui-quadrado.

+ valor de p do teste exato de Fisher.

entes, alguns tipos de alimentos podem não ser tolerados, representando significante risco de alterações do estado nutricional ${ }^{11,12}$.

O conhecimento do padrão alimentar a longo prazo,

pode alterá-la.

A cirurgia de derivação gástrica com bandagem em Y-de-Roux, é considerada excelente opção para perda do excesso de peso, resolução das comorbidades e manutenção da perda de peso $^{9,10}$. No entanto, pela restrição imposta aos pacide pacientes submetidos à cirurgia bariátrica, torna-se necessário para orientação e prevenção de distúrbios nutricionais. Alguns estudos sobre padrão alimentar pós-cirurgia bariátrica foram realizados por recordatório alimentar quantificando

Tabela 3 - Freqüência de vômitos espontâneos e induzidos após cirurgia.

\begin{tabular}{lccccccr}
\hline \multirow{2}{*}{ Tipo de Vômito } & \multicolumn{3}{c}{ Sexo } & \multicolumn{2}{c}{ Masculino } & \multicolumn{2}{c}{ Total } \\
\cline { 2 - 7 } & \multicolumn{2}{c}{ Feminino } & N & \% & n & \% \\
\hline Espontâneo & N & \% & 3 & 25,0 & 16 & 21,9 \\
Induzido & 13 & 21,3 & 9 & 75,0 & 57 & 78,1 \\
Total & 48 & 78,7 & 12 & 100,0 & 73 & 100,0 \\
\hline
\end{tabular}

Teste exato de Fisher: Valor de $p=0,7182$. 
macronutrientes consumidos diariamente e valor calórico da dieta com objetivo de estudar variações na perda de peso, ou comparar perda de peso conforme diferentes modificações na técnica cirúrgica, o que não foi a proposta desse estudo ${ }^{13,14}$.

O questionário foi formulado pelos autores baseado na experiência clínica, ele é simples de responder, fácil de preencher, reprodutível e pode permitir comparação ao longo do tempo com os pacientes individualmente e entre diferentes grupos de pacientes.

Optamos pela aplicação direta de um questionário simples, e elaborado conforme hábitos alimentares em nosso meio. Decidimos entrevistar diretamente o paciente ao invés de obter respostas por meio de cartas, telefonemas ou usando dados obtidos em registros de prontuário médico. A aplicação do questionário direto com o paciente nos proporcionou maior confiabilidade e menor perda de informações, além de ser aplicado sempre pela mesma pessoa, com conhecimento no assunto.

A população de gênero masculino e feminino foi semelhante demográfica e socialmente. Considerando o nível de escolaridade dos pacientes estudados, somente $6,5 \%$ não tiveram o $1^{\circ}$ grau concluído, as dificuldades com aceitação dos alimentos no pós-operatório, não deve ter sido por falta de entendimento das orientações pré-operatórias, mas sim pela dificuldade de adaptação à nova realidade. Nas consultas pré-operatórias os pacientes são orientados sobre a necessidade de modificação do modo habitual de se alimentar, principalmente sobre o tamanho das porções, à mastigação efetiva e ao tempo gasto por refeição. Uma maneira de alimentar-se mais é prolongar o tempo da refeição, cerca de vinte por cento deles aumentaram o tempo além de trinta minutos, tempo demasiadamente longo.

Comparamos os homens com as mulheres, pois há a impressão, nos atendimentos clínicos, que o padrão alimentar masculino é melhor que o feminino. Encontramos nos quesitos da aceitação alimentar, índices mais favoráveis às mulheres, exceto na aceitação de arroz e tamanho da redução das porções. Encontramos significância estatística apenas na aceitação de frutas que foi significativamente maior para as mulheres, tabela 2 .

A tolerância alimentar varia muito entre os pacientes submetidos à derivação gástrica com bandagem. Os alimentos mais frequientemente citados como de difícil aceitação são os de consistência fibrosa, seca e gordurosa como carnes, pão, arroz e vegetais crus ${ }^{15,17}$. Neste estudo os alimentos menos tolerados foram carne de boi, vegetais crus e arroz. A intolerância à carne é esperada devido à ressecção de grande parte do estômago com conseqüente alteração na produção de pepsina, principal responsável pela digestão das proteínas ${ }^{16}$. A carne bovina quando moída foi bem tolerada, pois sofrendo alteração nas fibras por ação mecânica facilita a digestão. Foi referido também melhor tolerância ao consumo de carne de porco ou frango. Quanto à dificuldade em aceitação de arroz, alimento habitual em nosso cardápio, a digestão é dificultada pelo processo de hidratação e gelatinização que o mesmo sofre quando submetido a cocção, dificultando a ação enzimática da amilase ${ }^{18}$.

Os vegetais, fontes de fibras, vitaminas e minerais foram bem tolerados quando bem cozidos. O feijão, alimento básico da nossa alimentação foi bem tolerado, amassado, com caldo ou inteiro. Contrário ao descrito em outros estudos, houve grande aceitação por pães ${ }^{15,18}$.

A intolerância alimentar, se intensa, pode ser a razão pela qual os pacientes passam a ingerir líquidos ou pastosos hipercalóricos, com efeito negativo sobre o emagrecimento.

Wardé-Kamar et al. ${ }^{19}$ estudaram o padrão alimentar de 69 pacientes após 4 anos de derivação gástrica com bandagem em Y-de-Roux e $62 \%$ de seus pacientes referiram vômitos espontâneos e $27 \%$ vômitos induzidos. Nosso estudo mostrou menor frequiência de vômitos tanto induzidos como espontâneos. Os vômitos espontâneos ocorreram em 21, 3\% das mulheres e 25\% dos homens, assim considerados se aconteceram mais que três vezes na semana (Tabela 3). Houve predominância dos vômitos em pacientes acima da sexta década, sem significância estatística. Os vômitos, na maioria das vezes, são decorrentes da ingestão de alimentos quantitativamente maiores que o estômago reduzido pode suportar e também por mastigação incorreta.

Estudos como este mostram a importância de revisões e companhamento em longo prazo, procurando fazer o paciente aderir às orientações para obter o resultado esperado quanto à perda e manutenção da perda de peso e prevenindo deficiências nutricionais. $\mathrm{O}$ acompanhamento de profissional de nutrição é fundamental no auxílio da equipe multiprofissional para garantir perda adequada de peso, sua manutenção, sem deficiências nutricionais.

As condições de realização do presente estudo permitem concluir que: com o questionário proposto foi possível avaliar o padrão tardio de aceitação alimentar dos pacientes e constatar que existe diferença entre homens e mulheres nas variáveis analisadas, apenas para o consumo de frutas.

\footnotetext{
ABSTRACT

Background: To evaluate late meal patterns in patients after Roux-en-Y gastric bypass surgery and to compare sex gender differences. Methods: From July 2006 to July 2007, patients from the outpatient Londrina Hospital State University - Digestive Surgery Section were evaluated after at least 12 months post-operatively. The patients were studied through a questionnaire, including demographic items about eating behavior, meal pattern, and frequency of vomiting and dumping symptoms. Statistical analysis was performed using Chi-square or Fischer test with significance requiring $p<0.05$. Results: Data were collected from 116 patients, 95(78.4\%) were women and $25(21.5 \%)$ were men. There were small differences when comparing women and men in eating behavior, meal pattern, frequency of vomiting and dumping symptoms and no statistical significance was found. Only the variables of fruits intake and somnolence were significantly more frequent in women. Conclusion: Applying the proposed questionnaire it was possible to know the late eating behavior and meal pattern of our patients. The intake of fruits was the unique item with statistical differences between genders.
}

Key words: Feeding; Obesity, morbid; Feeding behavior; Bariatric surgery; Gastric bypass; Food habits. 


\section{REFERÊNCIAS}

1. Thirlby RC, Randall J. A genetic "obesity risk index" for patients with morbid obesity. Obes Surg. 2002;12(1):25-9.

2. Quadros MRR, Savaris AL, Ferreira MV, Branco Filho A. Intolerância alimentar no pós-operatório de pacientes submetidos à cirurgia bariátrica. Rev Bras Nutr Clin. 2007;22:15-9.

3. Waitzberg DL. Nutrição oral, enteral e parenteral na prática clínica. $3^{\mathrm{a}}$ ed. v 1. São Paulo: Atheneu; 2000.

4. Pratt JS, Cummings S, Vineberg DA, Graeme-Cook F, Kaplan LM. Case records of the Massachusetts General Hospital. Weekly clinicopathological exercises. Case 25-2004. A 49-yearold woman with severe obesity, diabetes and hypertension. N Engl J Med. 2004; 351(70):696-705.

5. Silver HJ, Torquati A, Jensen GL, Richards WO. Weight, dietary and physical activity behaviors two years after gastric bypass. Obes Surg. 2006; 16(7):859-64.

6. Choban PS, Jackson B, Poplawski S, Bistolarides P. Bariatric surgery for morbid obesity: why, who, when, how, where, and then what? Cleve Clin J Med. 2002; 69(11):897-903.

7. MacLean LD, Rhode BM, Nohr CW. Late outcome of isolated gastric bypass. Ann Surg. 2000; 231(4):524-8.

8. Buchwald H, Avidor Y, Braunwald E, Jensen MD, Pories W, Fahrbach K, Schoelles K. Bariatric surgery: a systematic review and meta-analysis. JAMA. 2004; 292(14):1724-37.

9. Valezi AC, Mali Jr J, Brito EM, Marson AC. Gastroplastia vertical com bandagem em Y-de-Roux: análise de resultados. Rev Col Bras Cir. 2004; 31(1):49-56.

10. Garrido Jr. AB. Derivações gastrojejunais. In: Garrido Jr. AB. Cirurgia da Obesidade. $1^{a}$ ed. São Paulo: Atheneu; 2002. p.155-61.

11. Halverson J. Metabolic risk of surgery and long-term follow-up. Am J Clin Nutr. 1992;55(2 Suppl):602S-605S.

12. Faintuch J, Matsuda M, Cruz ME, Silva MM, Teivelis MP, Garrido AB Jr, Gama-Rodrigues JJ. Severe protein-caloric malnutrition after bariatric procedures. Obes Surg. 2004; 14(2):175-81.
13. Crampton NA, Izvornikov V, Stubbs RS. Silastic ring gastric bypass: a comparison of two ring sizes: a preliminary report. Obes Surg.1997; 7(6):495-9.

14. Olbers T, Björkman S, Lindroos A, Maleckas A, Lönn L, Sjöström L, Lönroth H. Body composition, dietary intake, and energy expenditure after laparoscopic Roux-en-Y gastric bypass and laparoscopic vertical banded gastroplasty: a randomized clinical trial. Ann Surg. 2006; 244(5):715-22.

15. Marcason W. What are the dietary guidelines following bariatric surgery? J Am Diet Assoc. 2004; 104(3):487-8.

16. Kenler HA, Brolin RE, Cody RP. Changes in eating behavior after horizontal gastroplasty and Roux-en-Y gastric bypass. Am J Clin Nutr.1990; 52(1):87-92.

17. Brolin RL, Robertson LB, Kenler HA, Cody RP. Weight loss and dietary intake after vertical banded gastroplasty and Roux-en-Y gastric bypass. Ann Surg. 1994; 220(6):782-90.

18. White S, Brooks E, Jurikova L, Stubbs RS. Long-term outcomes after gastric bypass. Obes Surg. 2005; 15(2):155-63.

19. Wardé-Kamar J, Rogers M, Flancbaum L, Laferrère B. Calorie intake and meal patterns up to 4 years after Roux-en-Y gastric bypass surgery. Obes Surg. 2004; 14(8):1070-9.

Como citar este artigo:

Valezi AC, Brito EM, Mali Jr J, Brito SJ. Estudo do padrão alimentar tardio em obesos submetidos à derivação gástrica com bandagem em y- de- Roux: comparação entre homens e mulheres. Rev Col Bras Cir. [periódico na Internet] 2008; 35(6). Disponível em URL: http:// www.scielo.br/rcbc

Endereço para correspondência:

Antônio Carlos Valezi

Rua Santos, 777 - apto. 1302

86020-041 - Londrina - PR

E-mail:valezi@sercomtel.com.br 